\title{
Correction to: A quantified study of segmentation techniques on synthetic geological XRM and FIB-SEM images
}

\section{Matthew Andrew ${ }^{1}$}

Published online: 21 September 2018

(C) Springer Nature Switzerland AG 2018

\section{Correction to: Comput Geosci \\ https://doi.org/10.1007/s10596-018-9768-y}

The original version of this article unfortunately contained mistakes introduced during the production process. The corrections are given in the following list:

(1) Figure 6 was missing. The figure is now given below:
(2) The last sentence of the 5th paragraph under Section 3 should be rewritten as:

The relative capacity of the different segmentation to classify based on texture can be seen by examining the fraction of misclassified voxels within the textural contrast phase (Fig. 6).

The original article has been corrected.
Fig. 6 Voxel misclassifications within the textural contrast region

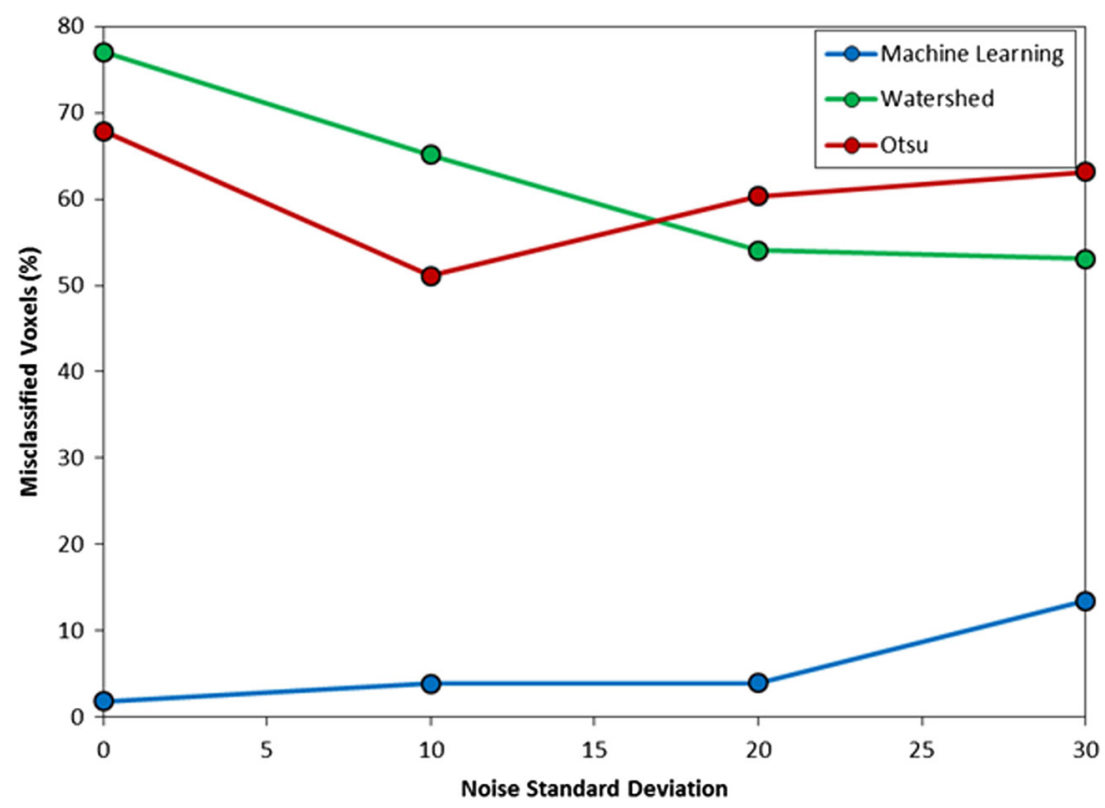

The online version of the original article can be found at https://doi.org/10.1007/s10596-018-9768-y.

Matthew Andrew

matthew.andrew@zeiss.com

1 Carl Zeiss Microscopy, 4385 Hopyard Rd, Pleasanton, CA, USA 